\title{
artigo
}

Santos, J.M.; Souza, J.F.; Ribeiro, C.L.; Esmeraldo, J.D.; Nascimento, S.M.M.; Nascimento, P.A.C.

Fatores de risco para a depressão infantil

\section{Fatores de risco para a depressão infantil}

\author{
Risk factors for child depression
}

Factores de riesgo de depresión infantil

\begin{abstract}
RESUMO
Depressão é uma condição associada à rebaixamento de humor, que tenha duração maior que duas semanas. Objetivo: Investigar através da literatura os fatores de risco que promovem o desenvolvimento de depressão em crianças. Método: Trata-se de uma Revisão Integrativa, realizada com 6 artigos, com os seguintes descritores: Depressão. Criança. Fatores de risco, articulado com o operador booleano AND, na LILACS, com corte temporal dos últimos cinco anos. Resultados: Os principais conteúdos abordados estão relacionados aos sintomas da depressão infantil e aos fatores causais da doença. À vista disso, identificou-se duas categorias baseadas nesses conteúdos, assim organizadas: Depressão na infância e os fatores e Ambientes que predispõem seu desenvolvimento e Sintomatologia habitual em crianças com transtorno depressivo. Conclusão: Apesar da quantidade limitada de artigos encontrados na literatura, a pesquisa conseguiu alcançar o propósito almejado, proporcionando uma discussão acerca dos fatores de riscos e dos sintomas da depressão no público infantil.
\end{abstract}

DESCRITORES: Depressão; Criança; Fatores de risco.

\section{ABSTRACT}

Depression is a condition associated with lowering mood, which lasts for more than two weeks. Objective: To investigate through the literature the risk factors that promote the development of depression in children. Method: This is an Integrative Review, carried out with 6 articles, with the following descriptors: Depression. Kid. Risk factors, articulated with the Boolean operator AND, at LILACS, with a time cut of the last five years. Results: The main specific contents are related to the symptoms of childhood depression and the factors that cause the disease. In view of this, two categories were identified based on the content, thus organized: Depression in childhood and the factors and Environments that predispose its development and Habitual symptoms in children with depressive disorder. Conclusion: Despite the limited amount of articles found in the literature, the research managed to achieve the desired purpose, providing a discussion about the risk factors and symptoms of depression in children. DESCRIPTORS: Depression; Children; Risk factors.

\section{RESUMEN}

La depresión es una condición asociada a la disminución del estado de ánimo, que dura más de dos semanas. Objetivo: Investigar a través de la literatura los factores de riesgo que favorecen el desarrollo de la depresión en los niños. Método: Se trata de una Revisión Integrativa, realizada con 6 artículos, con los siguientes descriptores: Depresión. Niño. Factores de riesgo, articulados con el operador booleano AND, en LILACS, con un corte de tiempo de los últimos cinco años. Resultados: Los principales contenidos específicos están relacionados con los síntomas de la depresión infantil y los factores causantes de la enfermedad. Ante esto, se identificaron dos categorías en función del contenido, así organizado: Depresión en la infancia y los factores y Ambientes que predisponen su desarrollo y Síntomas habituales en niños con trastorno depresivo. Conclusión: A pesar de la limitada cantidad de artículos encontrados en la literatura, la investigación logró alcanzar el propósito deseado, proporcionando una discusión sobre los factores de riesgo y síntomas de depresión en los niños.

DESCRIPTORES: Depresión; Niño; Factores de riesgo.

RECEBIDO EM: 28/02/2021 APROVADO EM: 08/03/2021

\section{Jessica Mota dos Santos}

Faculdade de Medicina Estácio de Juazeiro do Norte - Graduanda em Enfermagem.

ORCID: 0000-0003-3669-2048 


\section{Joice Fabrício de Souza}

Universidade de Fortaleza - Mestranda em Saúde Coletiva - PPGSC/UNIFOR. Bolsista pela Fundação Cearense de Apoio ao Desenvolvimento Científico e Tecnológico- FUNCAP. Integrante do Laboratório de Psicopatologia e Clínica Humanista Fenomenológica- APHETO, do Núcleo de Fenomenologia e Saúde - NUFES e do Grupo de Pesquisa Clínica, Cuidado e Gestão em Saúde - GPCLIN/URCA.

ORCID: 0000-0002-3165-1135

\section{Camila Lima Ribeiro}

Universidade de Fortaleza. Mestranda em Saúde Coletiva- PPGSC/UNIFOR. Especialista em Ginecologia e Obstetrícia - UNIQ; Especializanda em Enfermagem Forense - Unyleya.

ORCID: 0000-0002-1599-8454

\section{Joana D'arc Esmeraldo}

Mestre em Saúde da Criança e do Adolescente (UECE), Especialista em Política e Planejamento Educacional (URCA), Psicóloga (UNILEÃO), Graduação em Ciências Biológicas (URCA), Docente dos cursos de Enfermagem, Medicina e Psicologia da Faculdade de Medicina Estácio/FMJ, Coordenadora do Núcleo de Apoio ao Estudante de Medicina (NAEM) da Faculdade de Medicina Estácio/FMJ.

ORCID: 0000-0002-7578-8686

\section{Suiane Maria Mendes do Nascimento}

Enfermeira pela UniLeão. Especialista em UTI neonatal e UTI pediátrica, Capacitada em inserção de cateter PICC pelo Instituto AMA em SP. Especialista em saúde pública e da família. Mestrando em Cuidados Intensivos pelo IMBES. Preceptora de estágios da Faculdade de Medicina Estácio de Sá do Juazeiro do Norte.

ORCID: 0000-0002-0105-7442

\section{Polyana Amorim Cruz Nascimento}

Enfermeira Mestre em gestão em saúde pela universidade estadual do Ceará. Especialista em gestão da clínica e educação na saúde (Sírio Libanês), possui formação em saúde mental da infância e adolescência (Universidade Federal de Santa Catarina), Enfermeira efetiva do município de Mauriti e docente do curso de Medicina e enfermagem na Estácio FMJ. Possui formação em direito e saúde, Responsável pela criação e formulação do núcleo de conciliação nas defensorias públicas de Barbalha e Crato. ORCID: 0000-0002-9899-0932

\section{INTRODUÇÃO}

A depressão é um transtorno unipolar, sendo seu único polo a tristeza, ela atinge tanto adultos, como crianças e adolescentes. Este transtorno provém de inúmeras causas, desse modo, é importante uma investigação do contexto familiar, envolvendo fatores genéticos, do ambiente social em que o indivíduo está inserido e também das condições médicas relacionadas ${ }^{1}$.

A prevalência de crianças com transtornos mentais é de $1 \%$ a $20,3 \%$ no Brasil, e que uma entre cinco crianças e adolescentes desenvolve este tipo de distúrbio na população mundial. Segundo informações colhidas, de vinte crianças abaixo dos 10 anos de idade, uma evolui com um quadro de depressão e isso pode chegar a prejudicá-las no ambiente escolar e em vários outros ambientes em que o mesmo está inserido ${ }^{2,1}$.

A depressão é de difícil diagnóstico na infância, pois os sintomas desse transtor- no nos adultos se diferem nas crianças e algumas de suas características são atípicas e individuais. A criança pode apresentar oscilação de humor, desenvolver transtornos alimentares, dificuldades de concentração e aprendizagem em tarefas escolares, pode isolar-se e negar-se a brincar com outras crianças, provavelmente terá alteraçóes no sono, mostrará falta de interesse ao realizar atividades de lazer e em alguns casos é possível que ela venha a apresentar sintomas físicos como dor de cabeça e dor de barriga ${ }^{3}$.

É de suma importância que o diagnóstico seja feito precocemente, para tão logo iniciar o tratamento, pois, reduz o risco da criança desenvolver danos em seu amadurecimento. Para a realização do diagnóstico, faz-se necessário a investigação minuciosa de todo o histórico da doença, como a duração dos sintomas e o grau de comprometimento psicossocial, e além dessas informações colhidas, realizar o exame do estado mental da criança é primordial. $\mathrm{O}$ tratamento consiste em psicoterapia e farmacoterapia em casos mais grave $e^{4}$.

Dessa forma, é essencial conseguir identificar sintomas nas crianças e principalmente detectar o que provoca o início desses sintomas, para regredir a expansão descontrolada da doença. Assim é possível evitar que a criança acometida com o transtorno tenha um agravamento do quadro e pratique automutilação ou tentativas de suicídio no futuro. Diante o exposto, surge o seguinte questionamento para direcionamento da pesquisa: Quais são os fatores de risco que podem promover o desenvolvimento do transtorno depressivo em crianças?

O presente estudo tem como objetivo investigar através da literatura os fatores de risco que promovem o desenvolvimento do quadro depressivo no público infantil.

\section{MÉTODOS}

Trata-se de uma Revisão Integrativa da 
Literatura, portanto, o estudo é do tipo exploratório, descritivo e de natureza bibliográfica. Esta revisão integrativa de literatura, foi obtida por resultados de pesquisas que envolvem o tema do estudo, sendo constituída de forma ampla, ordenada e sistemática. Oferece informações mais holistas em concordância à problemática, sendo assim estabelecido um amplo conhecimento5.

Realizou-se uma busca por artigos que abordem os fatores de risco que promovem o desenvolvimento do quadro depressivo no público infantil. O período de elaboração do estudo iniciou-se no mês de janeiro de 2021 e estendeu-se até o início do mês de fevereiro do ano corrente, sendo analisados artigos científicos publicados em bases de dados. Feito a coleta dos artigos, a seleção foi feita através de seis etapas, e ao final de cada etapa foram excluídos os artigos que não atendiam aos critérios exigidos na pesquisa.

Os artigos selecionados para o presente estudo, foram buscados de forma pareada na Literatura Latino-americana e do Caribe em Ciências da Saúde (LILACS). Foram utilizados os seguintes descritores: "Fatores de risco", "Depressão" e "Criança”, as combinações entre os descritores foram cruzadas com o operador booleano "AND". Como critérios de inclusão optou-se por artigos na íntegra, online, publicados nos idiomas português, inglês

Figura 1: Fluxograma representando o processo de coleta de dados, Juazeiro do Norte, Ceará, Brasil ${ }^{6}$.

Estudo identificados (n=1306)

Busca na base de dados (LILACS)

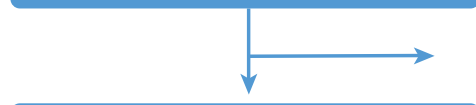

Estudos selecionados ( $\mathrm{n}=233$ )

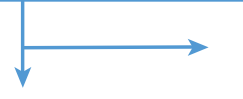

Estudos incluidos para sintese no estudo $(n=6)$

Estudos excluídos após leitura minuciosa $(n=227)$ e espanhol, grátis publicados nos últimos 5 anos (2016 a 2021) e que discutiam a temática proposta nos mais variados métodos de estudo. Excluiu-se estudos de natureza bibliográfica, teses, Trabalhos de Conclusão de Curso, dissertações, dossiês ou cartas ao autor e aquelas cujo método mão era rigorosamente descrito.

Com base em Mendes, Silveira e Galvão (2008) seguiu-se seis passos para a construção da Revisão Integrativa da Literatura: $1^{\text {a }}$ etapa: Será feito a leitura do título e do resumo; $2^{a}$ etapa: Será identificada a pergunta norteadora da temática, sendo selecionadas as hipóteses; $3^{a}$ etapa: Será realizada a leitura da metodologia e do resultado dos artigos;

$4^{a}$ etapa: Se fará uma leitura aprofundada do resultado; $5^{\text {a }}$ etapa: Delimitar os dados à serem utilizados dos estudos selecionados; $6^{a}$ etapa: Terá uma revisão dos artigos para a síntese de conhecimentos, onde será elaborado um resumo do resultado principal.

Após o cumprimento das etapas de coleta de dados dos artigos, uma tabela foi montada apresentando tópicos específicos, a fim de expor os dados mais importantes e classificatórios de cada artigo selecionado. A referida tabela apresentará os artigos que contenham os seguintes itens: autores, ano de publicação, objetivo do estudo, tipo de estudo, população ou amostra estudada e principal resultado.
Tratando-se de uma revisão integrativa da literatura, este estudo contém apenas dados secundários, não tendo envolvimento de seres humanos, portanto, não há necessidade de aprovação do Comitê de Ética e Pesquisa. As informações obtidas no estudo foram constatadas, respeitando a origem dos autores e de seus respectivos dados.

\section{RESULTADOS}

No período de levantamento de dados, utilizou-se descritores extraídos do Descritores em Ciências da Saúde (DeCS), obtendo-se um total de 1306 artigos, aplicando-se os filtros de recorte temporal e idiomas, adquiriu-se um total de 198 artigos, entretanto excluiu-se 192, dentre eles 165 por título e resumo, 15 revisão de literatura, $7 \mathrm{du}$ plicados e 5 fugiam da temática proposta.

Para a organização e apresentação das evidências encontradas, os dados foram expostos em um quadro, com a distribuição dos artigos segundo os autores, ano de publicação, tipo de estudo, objetivo e resultados.

De acordo com a análise feita na literatura, houve dificuldades para encontrar os artigos com os critérios exigidos para a pesquisa, pois há uma quantidade pequena de trabalhos publicados que abordem a temática proposta, tornando-se limitado o atual estudo, mas, mesmo com essa dificuldade foi possível dar continuidade à pesquisa, onde o tipo de estudo prevalente foi o transversal. Os principais conteúdos abordados estão relacionados aos sintomas da depressão infantil e aos fatores causais da doença.

À vista disso, identificaram-se duas categorias baseadas nesses conteúdos, assim organizadas: Depressão na infância e os fatores que predispõem seu desenvolvimento e Sintomatologia habitual em crianças com transtorno depressivo.

\section{DISCUSSÃO}

Categoria l: Depressão na infância e os fatores e ambientes que predispõem seu desenvolvimento

Nos anos 70, a depressão infantil era 
desconhecida para a maioria dos indivíduos e tida como uma doença rara, mais tarde, no ano de 1975 o Instituto Nacional de Saúde Mental dos Estados Unidos (NIMH) reconheceu o transtorno oficialmente, e, a partir daí, surgiu-se um interesse maior sobre o assunto entre profissionais de saúde e pesquisadores. Porém, apesar de muitas pesquisas relacionadas ao transtorno, o conceito do mesmo ainda é um pouco vago, devido a manifestação da doença ser diferente nas crianças e varian- do seus sintomas de acordo com cada fase da infância .

A família e a escola possuem deveres fundamentais no desenvolvimento infantil. Ambos em conjunto partilham atribuições educacionais, sociais e políticas, onde

\section{Quadro I: Organização dos artigos encontrados de acordo com o ano, autores, tipo de estudo, objetivo e resultados,} Juazeiro do Norte, Ceará, Brasil, 2021.

\begin{tabular}{|c|c|c|c|c|}
\hline $\mathbf{N}^{\circ}$ & $\begin{array}{c}\text { AUTORES/ } \\
\text { ANO }\end{array}$ & TIPO DE ESTUDO & OBJETIVO & RESULTADOS \\
\hline 1 & $\begin{array}{l}\text { Lima; } \\
\text { Pacheco, } \\
2018\end{array}$ & Estudo exploratório & $\begin{array}{c}\text { Investigar a associação entre indica- } \\
\text { dores de depressão, autorregulação } \\
\text { emocional e percepção de suporte } \\
\text { familiar }\end{array}$ & $\begin{array}{l}\text { As pesquisas sugerem que crianças com } \\
\text { depressão não empregam de maneira eficiente } \\
\text { estratégias de regulação afetiva e que quanto } \\
\text { maior a sintomatologia de depressão, menor o } \\
\text { suporte familiar percebido. }\end{array}$ \\
\hline 2 & $\begin{array}{c}\text { Aguiar et al, } \\
2018\end{array}$ & $\begin{array}{l}\text { Estudo quantitativo de } \\
\text { abordagem descritiva } \\
\text { comparativa }\end{array}$ & $\begin{array}{c}\text { Identificar e comparar as } \\
\text { características sociodemográficas de } \\
\text { famílias que buscaram atendimento } \\
\text { em um serviço-escola de Psicologia } \\
\text { e em um ambulatório de Psiquiatria } \\
\text { Infantil. }\end{array}$ & $\begin{array}{l}\text { As crianças atendidas na Psiquiatria obtiveram } \\
\text { mais escores clínicos para queixas somáticas, } \\
\text { problemas de pensamento e problemas } \\
\text { externalizantes. A comparação do perfil } \\
\text { das responsáveis indicou que a escolha da } \\
\text { modalidade de tratamento infantil pode estar } \\
\text { relacionada às variáveis maternas, sendo que } \\
\text { as mães com maior adversidade e depressão } \\
\text { optam pelo tratamento medicamentoso. }\end{array}$ \\
\hline 3 & $\begin{array}{c}\text { Órgiles et al, } \\
2018\end{array}$ & Estudo transversal & $\begin{array}{l}\text { Examinar a relação entre queixas } \\
\text { somáticas e sintomas depressivos } \\
\text { em crianças espanholas, levando em } \\
\text { consideração o sexo e a idade dos } \\
\text { participantes. }\end{array}$ & $\begin{array}{l}\text { Verificou-se que sintomas pseudoneurológicos, } \\
\text { dor e gastrointestinal são mais comuns em } \\
\text { crianças com sintomas depressivos, sendo a } \\
\text { dor nas articulações, cefaleia e fadiga os sinto- } \\
\text { mas mais prevalentes. }\end{array}$ \\
\hline 4 & $\begin{array}{l}\text { Baptista; } \\
\text { Borges; } \\
\text { Serpa, } 2017\end{array}$ & Estudo transversal & $\begin{array}{c}\text { Avaliar as diferenças na expressão dos } \\
\text { sintomas depressivos por gênero e faixa } \\
\text { etária. }\end{array}$ & $\begin{array}{l}\text { Evidenciou-se que as crianças apresentaram } \\
\text { mais medo da separação e maiores sentimen- } \\
\text { tos de solidão. }\end{array}$ \\
\hline 5 & $\begin{array}{l}\text { Oliveira; } \\
\text { Resende, } \\
2016\end{array}$ & $\begin{array}{l}\text { Pesquisa de campo, } \\
\text { do tipo descritiva e } \\
\text { comparativa }\end{array}$ & $\begin{array}{l}\text { Investigar sintomas depressivos e com- } \\
\text { portamentais em crianças em situação } \\
\text { de acolhimento institucional, por meio } \\
\text { do CDI e do CBCL. }\end{array}$ & $\begin{array}{c}\text { Os resultados apontam que as crianças do } \\
\text { GI (Grupo Instituição) apresentaram no CBCL } \\
\text { (Inventário de Comportamentos para Crian- } \\
\text { ças e Adolescentes entre } 6 \text { a } 18 \text { anos) média } \\
\text { de pontuação significativamente maior na } \\
\text { escala de problemas externalizantes (M=88,78; } \\
\text { DP=19,71) e no CDI (Inventário de Depressão } \\
\text { Infantil), média de pontuação significativamente } \\
\text { maior de sintomas depressivos ( } \mathrm{M}=12,61 ; \mathrm{DP} \\
=6,28) .\end{array}$ \\
\hline 6 & $\begin{array}{c}\text { Antunes et } \\
\mathrm{al}, 2016\end{array}$ & Estudo de caso & $\begin{array}{l}\text { Analisar os processos e as motivações } \\
\text { que levaram os pais e responsáveis } \\
\text { à percepção da depressão em suas } \\
\text { crianças e à consequente procura de } \\
\text { cuidado especializado. Busca também, } \\
\text { compreender a influência das crenças } \\
\text { em saúde e dos hábitos culturais na } \\
\text { procura de tratamento nestes casos. }\end{array}$ & $\begin{array}{l}\text { Notou-se existir uma ligação diretamente pro- } \\
\text { porcional da depressão com causas psicológicas } \\
\text { vividas a partir de experiências intrafamilia- } \\
\text { res. Nota-se também que os educadores são } \\
\text { profissionais que acompanham estas crianças } \\
\text { diariamente em suas atividades pedagógicas, } \\
\text { podendo perceber alterações aparentes de } \\
\text { comportamento ou desempenho escolar que } \\
\text { sinalizariam problemas mais sérios. }\end{array}$ \\
\hline
\end{tabular}


um tem o papel de complementar o outro, uma vez que, na escola o aprendizado torna-se mais estruturado e sistematizado, e na família o conhecimento repassado é mais informal, desse modo, promovendo a aprendizagem em grupos e individual. "A família é a primeira formação da criança”, pois é onde ela aprende os primeiros conceitos da vida, portanto esse ambiente deve ser de direitos, afeto e segurança. Os pais devem ser participativos na vida dos filhos, o que irá fazê-lo sentir-se valorizado, em consequência disso, desenvolve-se sua autoconfiança e sua autoestima, o que refletirá na vida adulta, tornando-se uma pessoa segura, autoconfiante e sabendo conviver em sociedade ${ }^{8}$.

A interação e os vínculos da família com a criança são de grande importância, visto que o contexto familiar é a fonte referencial da mesma, é nesse ambiente onde será intensificado o início de seu desenvolvimento, e ela irá absorver seus primeiros ensinamentos, sendo assim, definidos os primeiros vínculos afetivos. Os pais precisam estar atentos aos sinais que seus filhos demonstram, e intervir de acordo com a necessidade da criança?.

Por outro lado, é imprescindível que o indivíduo tenha uma boa educação infantil, pois é uma fase de muitas descobertas e de evolução de habilidades, na qual, irá garantir seu pleno desenvolvimento ${ }^{16}$. E para uma boa educação infantil é necessário a presença de profissionais capacitados para o cargo. O professor em sua atuação com o público infantil, precisa conhecer a história de cada um dos seus alunos, exercer interação com suas famílias, mantendo diálogo com as mesmas, observar todo o processo educativo, e as pessoas que o envolve, desse modo, possuindo formação nas áreas técnica, política, ética, estética e psicológica ${ }^{10}$.

É essencial dar atenção nesses ambientes em que a criança está inserida, pois eles irão definir o desenvolvimento adequado ou deficiente da mesma. Portanto se a criança vive em um ambiente desfavorável e deficiente para seu desenvolvimento, ela tem grandes probabilidades de desenvolver qualquer doença, e uma delas pode ser a depressão ${ }^{11}$.
Qualquer tipo de instabilidade, traumas, negligências, fragilidades, desequilíbrios, pode ser um fator de risco para atrapalhar o progresso de sua evolução, e assim, ser capaz de intensificar condiçõos preexistentes ou desencadear novos distúrbios ${ }^{12}$.

A criança pode vir a desenvolver o transtorno depressivo após acontecimentos de luto ou perda, como separação dos pais ou óbito de entes queridos. Posteriormente, a mesma começa a apresentar mudanças em seu comportamento, e o agravamento da patologia se dá pela incompreensão dos pais em relação à situação do filho, pois desconhecem a sintomatologia da doença ${ }^{13}$.

O grau de instrução dos genitores, está ligado à condição financeira dos mesmos, e pesquisas indicam que quanto menor o grau de instrução dos pais, menor será sua capacidade de instruir e relacionar-se com seus filhos, trazendo riscos para que a criança desenvolva o transtorno ${ }^{14}$.

\section{Categoria II: Sintomatologia habitual em crianças com transtorno depressivo}

O transtorno depressivo tem o poder de causar degradação do processo de maturidade social e psicológica da criança e comprometer drasticamente seu desenvolvimento. Crianças não apresentam sintomas característicos iguais aos do indivíduo adulto, nela, eles irão se manifestar de acordo com cada fase infantil, como exemplo, ela pode apresentar um aumento da sensibilidade ou pessimismo, o que por sua vez, é considerado por alguns pesquisadores como sintomas "mascarados", logo, o transtorno pode ser confundido com outras patologias como o Déficit de Atenção e/ou Hiperatividade $(\mathrm{TDAH})^{15}$.

O paciente que apresenta sintomas de depressão, pode manifestar episódios maníacos e depressivos. Na ocorrência dos episódios depressivos a criança apresenta um autojulgamento exagerado, sente-se culpada, envergonhada e não se considera merecedora de ser feliz. Já na manifestação da mania, o paciente torna-se agitado, inquieto, e, na maioria das vezes apresenta grande agitação psicomotora ${ }^{16}$.

Há um tipo de depressão leve e um tipo da doença mais profunda. $\mathrm{Na}$ síndrome leve o indivíduo ainda consegue realizar suas atividades cotidianas, porém, sente-se fadigado, infeliz, desanimado, com sentimentos pessimistas e sempre entediado, não demonstrando nenhuma reação. Já no tipo mais profundo, a criança sente-se desesperada, angustiada, dor mental, sentimentos profundos de medo e raiva, faz críticas à sua existência e demonstra desejo de morte ${ }^{17}$.

Um dos principais sintomas da presença do quadro depressivo na criança e algo imprescindível para detecção desse transtorno, é o retrocesso no desempenho escolar, pois a criança começa a ter falta de interesse e dificuldade para se concentrar nos conteúdos. Dessa forma, em junção com outros fatores agravantes como dores físicas e fobias específicas, a criança pode isolar-se, recusar-se a ir à escola e ter dificuldades de relacionamento com os colegas ${ }^{7}$.

A principal manifestação clínica nas crianças de até 6 anos são sintomas somáticos, tal como dores abdominais, fadiga, cefaleia e tontura e essas queixas, na maioria das vezes, são acompanhadas de diminuição do apetite, perda de peso, irritabilidade, alterações de sono e agitação psicomotora. Alguns sintomas atípicos incluem enurese, encoprese, comunicação deficiente, agressividade e movimentos repetitivos ${ }^{18}$.

Sobre o suicídio infantil, há poucos estudos publicados sobre o assunto, porém, dados do Ministério da Saúde confirmam que as taxas desse fenômeno estão aumentando cada vez mais no Brasil, $40 \%$ dessa taxa aumentou de 2002 a 2012 em crianças e pré-adolescentes com faixa etária de 10 a 14 anos. É importante observar os principais sintomas e procurar escutar a criança, estar sempre vigiando o uso da internet, não ter preconceitos com internações, pois assim pode-se evitar o suicídio na infância ${ }^{19}$.

\section{CONCLUSÃO}

Apesar da quantidade limitada de artigos encontrados na literatura, a pesquisa alcançou o propósito almejado, proporcionando uma discussão acerca dos fatores de riscos e dos sintomas da depressão no público infantil. Nota-se a importância do 
trabalho em conjunto da família e da escola, tanto para identificação de possíveis sintomas da depressão, quanto na prevenção da doença, pois é extremamente complicado o diagnóstico nas crianças, devido aos sintomas inespecíficos e também pela criança não saber relatar seus sentimentos. Além desses ambientes serem essenciais, principalmente no tratamento da criança acometida pelo distúrbio, pois sendo um ambiente favorável, há a possibilidade da melhora da doença ou até cura, e sendo um ambiente desfavorável, podem haver consequências irreversíveis e levar a um quadro mais grave deste distúrbio.

\section{REFERÊNCIAS}

1. Guerra MGGV, Almeida FMM, Afonso DB. Depressão Infantil: ensino-aprendizagem a partir de uma experiência escolar no espírito santo. Revista Cesumar - Ciências Humanas e Sociais Aplicadas [Internet]. 2018 [cited 2021 Jan 6];23(1):77-94. Available from: https://periodicos.unicesumar.edu.br/index.php/revcesumar/article/view/6508.

2. Leite LML. Eventos de vida estressantes vivenciados pelas mães e depressão infantil [Dissertação on the Internet]. Recife: Universidade Federal de Pernambuco; 2019 [cited 2021 Jan 6]. Available from: https://repositorio.ufpe.br/handle/123456789/34336 Pós-graduação em Saúde Coletiva.

3. Schimidtt BG. Sofrimento psíquico e depressão na infância: o acolhimento da criança como sujeito a ser escutado [Trabalho de Conclusão de Curso on the Internet]. ljuí: Universidade Regional do Noroeste do Estado do Rio Grande do Sul; 2019 [cited 2021 Jan 8]. 12 p. Available from: https://bibliodigital.unijui.edu.br:8443/xmlui/ handle/123456789/6571 Psicologia.

4. Ercole FF, Melo LS; Alcoforado CLGC. Revisão integrativa versus revisão sistemática. Revista Mineira de Enfermagem, v. 18, n. 1, p. 9-12, 2014.

5. Alexandre KSM. Depressão Infantil [Trabalho de Conclusão de Curso on the Internet]. [place unknown]: Centro Universitário do Planalto Central Apparecido dos Santos; 2018 [cited 2021 Jan 6]. 5 s. Available from: https://dspace.uniceplac.edu.br/handle/123456789/242 Odontologia.

6. Mendes KDS, Silveira RCCP, Galvão CM. Revisão integrativa: método de pesquisa para a incorporação de evidências na saúde e na enfermagem. Texto contexto - enfermagem, Florianópolis, v. 17, n. 4, p. 758-764, Dec. 2008.

7. Bordignon L, Gomes KM, Nunes RZS, Zavadil SC, Amboni G, Tomasi CD, Bitencourt LTG, Ceretta LB. Depressão Infantil e Dificuldades de Aprendizagem / Child Depression and Learning Difficulties. Id On Line Revista de Psicologia [Internet]. 2019 Oct 28 [cited 2021 Jan 6];13(47):327-342. Available from: https://idonline.emnuvens.com.br/id/article/view/1985.

8. Costa EL, Souza JRS. Família escola: as contribuições da participação dos responsáveis na educação infantil. Khora [Internet] 2019 [cited 2021 Jan 6];6(7):113-121. Available from: http://site. feuc.br/khora/index.php/vol/article/download/166/113.

9. Esteves LP, Ribeiro S. A importância dos vínculos afetivos e da interação familiar para a formação e aprendizagem escolar das crianças. Revista Psicologia, Diversidade e Saúde [Internet]. 2016 [cited 2021 Jan 6];5(2):206-214. Available from: https://www5. bahiana.edu.br/index.php/psicologia/article/view/879/771.

10. Silva S, Monteiro SS, Rodrigues MF. A importância da Educação Infantil para o pleno desenvolvimento da criança. Revista Mosa- ico [Internet]. 2017 [cited 2021 Jan 8];8(2):30-38. Available from: http://editora.universidadedevassouras.edu.br/index.php/RM/article/download/1170/pdf/.

11. Leite SRM, Carvalho AB. Relação entre a educação infantil e a família: em busca de uma educação acolhedora. Nuances [Internet]. 2018 [cited 2021 Jan 6];29(2):211-223. Available from: https://revista.fct.unesp.br/index.php/Nuances/article/ view/4561.

12. Dias FS. Depressão infantil no contexto escolar: construções possíveis a partir da percepção dos professores [Trabalho de Conclusão de Curso on the Internet]. Santa Cruz do Sul: Universidade de Santa Cruz do Sul; 2019 [cited 2021 Jan 6]. 7s. Available from: https://repositorio.unisc.br/jspui/handle/11624/2544 Psicologia.

13. Silva AKL, Silva TSF, Rodrigues LGF, Souza MO, Teixeira VPG. 0 impacto da negligência familiar no desenvolvimento infantil. GepNews [Internet]. 2018 [cited 2021 Jan 8];1(1):274-279. Available from: https://www.seer.ufal.br/index.php/gepnews/article/download/6392/4458.

14. Vieira GM. Perceções de Sintomatologia de Depressão Infantil em crianças do $1^{\circ}$ ciclo do ensino básico [Tese on the Internet] [place unknown]: Escola Superior de Educação de Paula Frassinetti; 2018 [cited 2021 Jan 8]. 95 s. Available from: http://hdl.handle. net/20.500.11796/2698 Mestrado em Ciências da Educação-especialização em Educação Especial.

15. Costa MHP. A influência da família e da escola no processo do desenvolvimento infantil. Argamassa [Internet]. 2018 [cited 2021 Jan 6];1(3):46-55. Available from: https://periodicos.ufms.br/index.php/argamassa/article/view/9062.

16. Sá LA. Depressão Infantil: elaboração de um instrumento para avaliação e tomada de decisão em saúde [Dissertação on the Internet]. João Pessoa: Universidade Federal da Paraíba; 2017 [cited 2021 Jan 8]. 106 s. Available from: https://repositorio.ufpb.br/ jspui/handle/tede/9044 Programa de Pós-graduação em Modelos de Decisão e Saúde.

17. Silva BM, Ferreira TA, Esper MV. Depressão na infância: olhar do psicopedagogo. Revista Amazônica [Internet]. 2019 [cited 2021 Jan 8];24(2):464-482. Available from: https://periodicos. ufam.edu.br/index.php/amazonica/article/view/6767/4763.

18. Santos SO, Maio APV, Barbosa CBB, Souza JM, Simões VAP. Depressão Infantil: sintomas e aspectos sociais, psicológicos na educação escolar. EDUCERE - Revista da Educação [Internet]. 2016 [cited 2021 Jan 8]; 16(1):47-60. Available from: https://revistas.unipar.br/index.php/educere/article/view/5824/3317.

19. Marconi EVN. Depressão Infantil: uma revisão bibliográfica. Psicologia.Pt [Internet]. 2017 [cited 2021 Jan 8]; 10(2):1-17. Available from: https://www.psicologia.pt/artigos/textos/A1091.pdf. 